\title{
Speckle interferometry and radiative transfer modelling of the Wolf-Rayet star WR 118
}

\author{
B. Yudin ${ }^{1}$, Y. Balega ${ }^{2}$, T. Blöcker ${ }^{3}$, K.-H. Hofmann ${ }^{3}$, D. Schertl ${ }^{3}$, and G. Weigelt ${ }^{3}$ \\ 1 Sternberg Astronomical Institute, Universitetskii pr. 13, 119899 Moscow, Russia \\ 2 Special Astrophysical Observatory, Nizhnij Arkhyz, Zelenchuk region, Karachai-Cherkesia, 35147, Russia \\ e-mail: balega@sao.ru \\ 3 Max-Planck-Institut für Radioastronomie, Auf dem Hügel 69, 53121 Bonn, Germany \\ e-mail: bloecker@mpifr-bonn.mpg.de; hofmann@mpifr-bonn.mpg.de; schertl@mpifr-bonn.mpg.de; \\ weigelt@mpifr-bonn.mpg.de
}

Received 16 July 2001 / Accepted 7 September 2001

\begin{abstract}
WR 118 is a highly evolved Wolf-Rayet star of the WC10 subtype surrounded by a permanent dust shell absorbing and re-emitting in the infrared a considerable fraction of the stellar luminosity. We present the first diffraction-limited $2.13 \mu \mathrm{m}$ speckle interferometric observations of WR 118 with 73 mas resolution. The speckle interferograms were obtained with the $6 \mathrm{~m}$ telescope at the Special Astrophysical Observatory. The twodimensional visibility function of the object does not show any significant deviation from circular symmetry. The visibility curve declines towards the diffraction cut-off frequency to $\sim 0.66$ and can be approximated by a linear function. Radiative transfer calculations have been carried out to model the spectral energy distribution, given in the range of $0.5-25 \mu \mathrm{m}$, and our $2.13 \mu \mathrm{m}$ visibility function, assuming spherical symmetry of the dust shell. Both can be fitted with a model containing double-sized grains ("small" and "large") with the radii of $a=0.05 \mu \mathrm{m}$ and $0.38 \mu \mathrm{m}$, and a mass fraction of the large grains greater than $65 \%$. Alternatively, a good match can be obtained with the grain size distribution function $n(a) \sim a^{-3}$, with $a$ ranging between $0.005 \mu \mathrm{m}$ and $0.6 \mu \mathrm{m}$. At the inner boundary of the modelled dust shell (angular diameter $\Theta_{\text {in }}=(17 \pm 1)$ mas), the temperature of the smallest grains and the dust shell density are $1750 \mathrm{~K} \pm 100 \mathrm{~K}$ and $(1 \pm 0.2) \times 10^{-19} \mathrm{~g} / \mathrm{cm}^{3}$, respectively. The dust formation rate is found to be $(1.3 \pm 0.5) \times 10^{-7} M_{\odot} / \mathrm{yr}$, assuming $V_{\text {wind }}=1200 \mathrm{~km} \mathrm{~s}^{-1}$.
\end{abstract}

Key words. techniques: image processing - circumstellar matter - stars: individual: WR 118 - stars: mass-loss stars: Wolf-Rayet - infrared: stars

\section{Introduction}

The Wolf-Rayet star WR 118 (= CRL $2179=$ IRAS 182891001) in the catalog of WR stars of van der Hucht (2001) belongs to the latest subtypes of the carbon-rich sequence (WC). It is classified as WC9-10 (Allen et al. 1977; Massey \& Conti 1983). Its very prominent IR excess is attributed to the thermal emission of a warm carbon dust shell. No remarkable variations in the dust emission have been registered over the last two decades (Williams et al. 1987; van der Hucht et al. 1996) and it has been designated as a "persistent" dust-maker (Williams \& van der Hucht 2000). While the dust grains are momentum-coupled to the fast stellar wind, fresh dust grains should be steadily condensing at a definite distance from the star, i.e. the dust formation rate is generally constant on the time scale of several decades.

Send offprint requests to: B. Yudin, e-mail: yudin@sai.msu.ru
Among the known galactic WR stars, WR 118 is one of the optically faintest objects, but one of the brightest ones in the infrared regime. This is naturally explained by its heavy interstellar reddening and the obscuration by its conspicuous dust shell. A strong interstellar $9.7 \mu \mathrm{m}$ silicate feature is observed in its spectrum with an optical depth of $\tau_{9.7 \mu \mathrm{m}} \approx 0.7$ (Roche \& Aitken 1984; van der Hucht et al. 1996). Using the relation $A_{V} / \tau_{9.7 \mu \mathrm{m}} \leq 21.5$ (Schutte et al. 1998), an extinction of $A_{V} \leq 15$ can be derived. Allen et al. (1977) and Williams et al. (1987) list $A_{V} \sim 15$ and $\sim 12.8$, respectively. In the optical region, the brightness of WR 118 is only roughly known.

From spectrophotometry, Massey \& Conti (1983) estimate the $v$ narrow-band magnitude to be approximately 22, and Cohen \& Vogel (1978) list a $V$ broad-band magnitude of $\sim 20$. Williams et al. (1987) modelled the IR spectral energy distribution (SED) of the WC stars, finding that they could be well fitted with an optically thin shell consisting of small $(\sim 0.01 \mu \mathrm{m})$ amorphous carbon grains 
with a density distribution of $\rho \propto r^{-2}$. The absence of the silicon carbide feature at $11.3 \mu \mathrm{m}$ in the spectra of WR stars exclude $\mathrm{SiC}$ to be a constituent of their dust shells. In this paper we present diffraction-limited 73 mas speckle interferometric observations of the dust shell of WR 118. Radiative transfer calculations have been performed to model both the SED and the $2.13 \mu \mathrm{m}$ visibility.

\section{Observations and data reduction}

The WR 118 speckle interferograms were obtained with the Russian $6 \mathrm{~m}$ telescope at the Special Astrophysical Observatory on September 27, 1999. The speckle data were recorded with our HAWAII speckle camera $(\mathrm{HgCdTe}$ array, $256^{2}$ pixels, sensitivity from 1 to $2.5 \mu \mathrm{m}$, frame rate 2 frames/s) through an interference filter with a central wavelength of $2.13 \mu \mathrm{m}$ and a bandwidth of $0.21 \mu \mathrm{m}$. Speckle interferograms of the unresolved star HIP 87540 were taken for the determination of the atmospheric speckle transfer function. The observational parameters were as follows: exposure time/frame $20 \mathrm{~ms}$; number of frames 210 (130 of WR 118 and 80 of HIP 87540; $2.13 \mu \mathrm{m}$ seeing $(F W H M)$ 1!! 4; field of view 5!! $1 \times 5$ !' 1 ; pixel size 26.4 mas. The resolution was 73 mas. The visibility function (modulus of the Fourier transform of the object intensity) was determined with the speckle interferometry method (Labeyrie 1970). Figure 1 shows the twodimensional $2.13 \mu \mathrm{m}$ visibility and the azimuthally averaged $2.13 \mu \mathrm{m}$ visibility of WR 118 up to the diffraction cut-off frequency. There is no evidence for deviation from circular symmetry. The visibility curve can be approximated by a linear function and dropped to 0.66 at the diffraction cut-off frequency.

\section{The dust shell model}

Using the quasi-diffusion method of Leung (1975), as implemented in the CSDUST3 code (Egan et al. 1988), we have constructed spherically symmetric radiative transfer models to match both the observed SED and the $2.13 \mu \mathrm{m}$ visibility. The input parameters of the model are: (i) the spectral shape and bolometric luminosity of the star $\left(f(\lambda) L_{\mathrm{bol}}\right)$, after passing the shell, $f(\lambda) L_{\mathrm{bol}}$ transforms to $f^{\prime}(\lambda) L_{\mathrm{bol}}$; (ii) the inner shell radius $\left(r_{\text {in }}\right)$; (iii) the relative thickness of the shell, i.e. the ratio of outer to inner shell radius $\left(Y_{\text {out }}=r_{\text {out }} / r_{\text {in }}\right)$; (iv) the density distribution $\rho(r)$; (v) the chemical composition and grain-size distribution; (vi) the total optical depth at a given reference wavelength.

The input parameter of the radiative transfer model, which relates the stellar bolometric luminosity and the inner shell radius, is the incident flux at the inner boundary of the dust shell: $F_{\text {in }}=L_{\text {bol }} /\left(4 \pi r_{\text {in }}^{2}\right)$. It is based on the assumption that the temperature of the dust grains is controlled by the radiation field only. This means that if we change $L_{\text {bol }}$ but keep $F_{\text {in }}=$ const. (i.e. change $r_{\text {in }} \propto \sqrt{L_{\text {bol }}}$, resp.) we obtain (i) the same SED; (ii) the
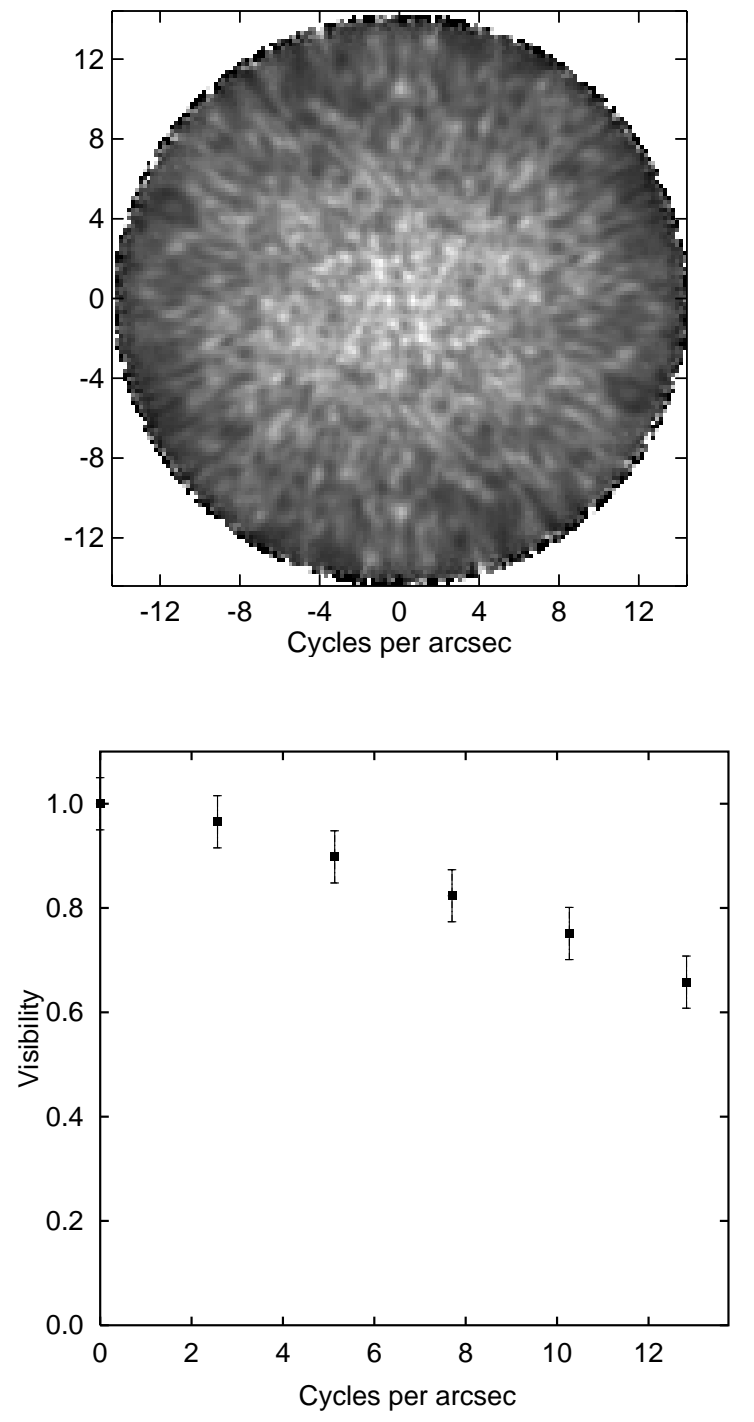

Fig. 1. Two-dimensional $2.13 \mu \mathrm{m}$ visibility (top) and azimuthally averaged $2.13 \mu \mathrm{m}$ visibility of WR 118 (bottom) shown up to the diffraction limit.

same normalized surface brightness distribution as a function of $b / r_{\text {in }}\left(b / r_{\text {in }}\right.$ : impact parameter); and (iii) the same visibility curve as function of $q \Theta_{\text {in }}$ ( $q$ : spatial frequency; $\Theta_{\text {in }}$ : angular diameter of the dust shell's inner boundary, $\left.b / 2 r_{\text {in }}=\Theta / \Theta_{\text {in }}\right)$. For more details of self-similarity and scaling behaviour of infrared emission from radiatively heated dust, see also Ivezic \& Elitzur (1997) and references therein. For the sake of clarity, we have incorporated $L_{\text {bol }}$ and $r_{\text {in }}$ separately in the CSDUST3 code instead of using $F_{\text {in }}$ only. During the SED fitting procedure, we kept $L_{\text {bol }}$ constant, i.e. changed only $r_{\text {in }}$.

Keeping in mind that the distance is defined by the relation $d=\sqrt{f^{\prime}(\lambda) L_{\mathrm{bol}} / 4 \pi F_{\mathrm{obs}}(\lambda)}\left(F_{\mathrm{obs}}(\lambda)\right.$ : observed (dereddened) spectral energy flux), i.e. $d \propto \sqrt{L_{\text {bol }}}$, it can be shown that the angular surface brightness distribution of the model, in particular $\Theta_{\text {in }}=2 r_{\text {in }} / d$, does not depend on $L_{\text {bol }}$. In our calculations $\Theta_{\text {in }}$ is determined by comparing the modelled and observed $K$-band $(\lambda=2.2 \mu \mathrm{m})$ flux. We note that, if the observed SED is properly fitted, we 
Table 1. Model dust-shell parameters. The models M1, M 2, M 1.1 and M 2.1 refer to double-sized grains with $a=0.05 \mu \mathrm{m}$ and $a=0.38 \mu \mathrm{m}$, the model M 3 to a grain size distribution of $n(a) \propto a^{-3}$ with $0.005 \mu \mathrm{m} \leq a \leq 0.6 \mu \mathrm{m}$. $\tau_{V}$ is the optical depth at $0.55 \mu \mathrm{m} ; r_{\text {in }}, T_{\text {in }}$ and $\Theta_{\text {in }}$ are the radius, the temperature (refering to the smallest grains) and the angular diameter of the inner dust shell rim, $n_{0.05}$ and $m_{0.05}$ are the abundances of $0.05 \mu \mathrm{m}$ grains by number and mass, resp., and $A_{K}$ is the $2.2 \mu \mathrm{m}$ dust-shell albedo.

\begin{tabular}{lccccccc}
\hline model & $\tau_{V}$ & $\begin{array}{c}r_{\text {in }} \\
\left(R_{*}\right)\end{array}$ & $\begin{array}{c}T_{\text {in }} \\
(\mathrm{K})\end{array}$ & $\begin{array}{c}n_{0.05} \\
(\%)\end{array}$ & $\begin{array}{c}m_{0.05} \\
(\%)\end{array}$ & $A_{K}$ & $\begin{array}{c}\Theta_{\text {in }} \\
(\mathrm{mas})\end{array}$ \\
\hline M 1 & 0.88 & 179 & 1670 & 99.55 & 34 & 0.36 & 17.8 \\
M 2 & 0.70 & 140 & 1820 & 98.83 & 17 & 0.40 & 16.3 \\
M 3 & 0.70 & 152 & 1760 & & & 0.36 & 17.5 \\
M 1.1 & 0.73 & 159 & 1740 & 99.55 & 34 & 0.36 & 17.4 \\
M 2.1 & 0.58 & 125 & 1900 & 98.83 & 17 & 0.40 & 16.0 \\
\hline
\end{tabular}

get the same value of $\Theta_{\text {in }}$ conducting the flux normalization at any other wavelength. In principle, the bolometric flux can be used as well, but for WR 118 this appears to be unfavorable due to the lack of optical and UV data.

In our model calculations, we firstly assumed a bolometric luminosity of $L_{\text {bol }}=4 \times 10^{4} L_{\odot}$. Luminosityindependent model parameters are given in Table 1. Removing the reddening from the observed $J$ magnitude of WR 118 and taking the model $V-J$ color, we obtain the (unreddened) model $V_{0}$ magnitude of WR 118 .

Adopting a WC9-10 absolute magnitude of $M_{V}=$ -4.9 (Williams et al. 1987), $d$ and $L_{\text {bol }}$ can be estimated. Taking this new value of $L_{\text {bol }}$ (see Table 2) we recalculated the luminosity-dependent model parameters such as $r_{\text {in }}$, the rate of dust formation at the inner boundary of the dust shell ( $\left.\dot{M}_{\text {dust }}\right)$, and the density of the dust shell at the inner boundary $\left(\rho_{\text {in }}\right)$ using the relations $r_{\text {in }} \propto \sqrt{L_{\text {bol }}}$, $\dot{M}_{\text {dust }} \propto \sqrt{L_{\text {bol }}}$, and $\rho_{\text {in }} \propto 1 / \sqrt{L_{\text {bol }}}$. Concerning the dust formation rate, an outflow velocity of $V_{\text {wind }}=1200 \mathrm{~km} \mathrm{~s}^{-1}$ was adopted (Williams et al. 1987). The model predictions for all these luminosity dependent parameters of WR 118 are given in Table 2 .

For the SED of the star, $f(\lambda)$, we took basically a black body of $19000 \mathrm{~K}$ (van der Hucht et al. 1986). We corrected it in the region of $0.5-1.25 \mu \mathrm{m}$ to have $f(\lambda) \propto \lambda^{-3.0}$ (Williams et al. 1998) corresponding to $(V-J) \approx 0.0$. In the infrared, corrections for the continuum radiation of the ionized gas shell have been made to obtain $(J-K) \approx 0.25$ and $f(\lambda) \propto \lambda^{-2.7}$ longwards of $10 \mu \mathrm{m}$ (Williams et al. 1987). It should be noted that these corrections have only minor effects on the model SED due to a very strong IR excess caused by the emission of dust grains.

The density distribution of the dust shell is given by $\rho \propto r^{-2}$, i.e. by a uniform outflow model. The outer shell boundary was chosen to be $Y_{\text {out }}=10^{3}$. Larger values of $Y_{\text {out }}$ affect the SED only in the far-infrared, i.e. far beyond the IRAS $25 \mu \mathrm{m}$ photometric band, which defines the long-wavelength tail in this study. Concerning the optical dust-grain constants, we considered the amorphous

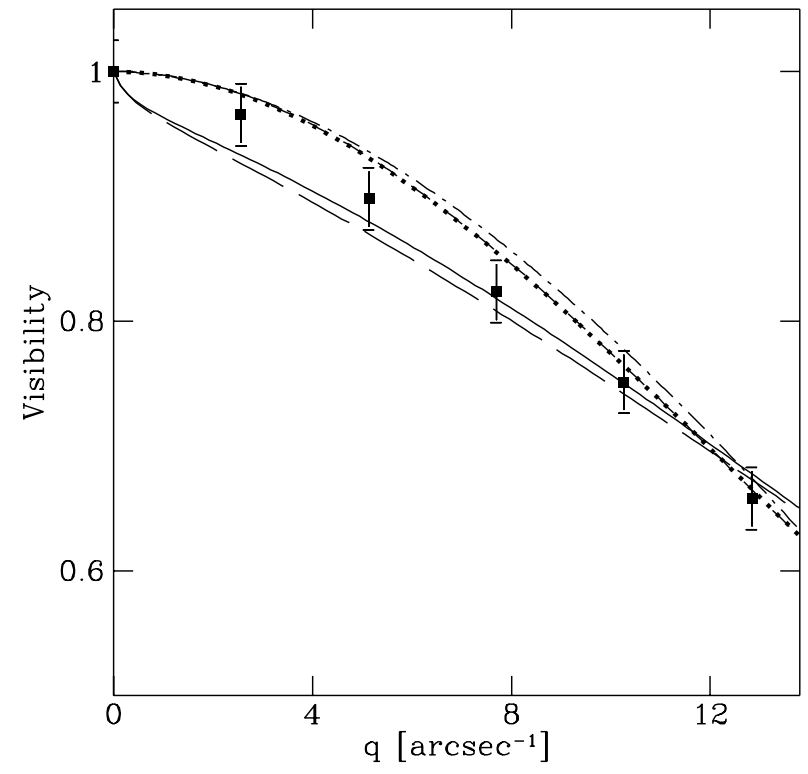

Fig. 2. $2.13 \mu \mathrm{m}$ model visibility function of WR 118 for different optical depths and grain sizes for a dust-shell with $r_{\text {in }}=200 R_{*}$ together with the observed visibility. The dotted, short-dashed, dot-dashed, solid and long-dashed lines refer to $\tau_{V}=0.6, a=0.01 \mu \mathrm{m} ; \tau_{V}=0.6, a=0.10 \mu \mathrm{m}$; $\tau_{V}=4.0, a=0.10 \mu \mathrm{m} ; \tau_{V}=4.0, a=0.38 \mu \mathrm{m}$; and $\tau_{V}=0.6$, $a=0.38 \mu \mathrm{m}$, resp.

carbon grains (cel1000) of Jäger et al. (1998). The reference wavelength of the optical depth was chosen to be $0.55 \mu \mathrm{m}$, and the model SED is normalized to the observed one in the $J$ band.

Figure 2 shows the $2.13 \mu \mathrm{m}$ model visibility for different optical depths (0.6 and 4) and grain sizes (single-sized grains with $0.01 \mu \mathrm{m}, 0.1 \mu \mathrm{m}$ and $0.38 \mu \mathrm{m}$ ) for a dust shell with $r_{\mathrm{in}} / R_{*}=200$ in comparison with the observed visibility. Only for the model with the largest grains, the visibility curve approaches to a linear function, i.e. its shape approaches the one of the observed visibility of WR 118 .

The transformation of the shape of the $2.13 \mu \mathrm{m}$ visibility curve from parabolic to rectilinear is connected with the transformation of a mostly thermally emitting dust shell to a highly scattering one when the albedo of dust grains is getting large. The $2.13 \mu \mathrm{m}$ albedo of the Jäger et al. (1998) carbon grains with $a=0.1 \mu \mathrm{m}$ is 0.04 , i.e. the dust shell's radiation is dominated by direct thermal emission at this wavelength. The albedo of the corresponding $0.38 \mu \mathrm{m}$ grains is 0.4 , i.e. the dust shell scatters the IR light very efficiently. Increasing the optical depth of an "emitting" and a "scattering" dust shell results into a slight increase and decrease of the visibility's curvature, respectively (Fig. 2).

Keeping in mind the very hostile environment for dust condensation in the shells of WR stars, it appears to be obvious to assume that the dust grains are rather small (Williams et al. 1987). However, such models will not be able to reproduce the shape of the visibility curve of WR 118. On the other hand, it is clear that the dust shell cannot consist entirely of large grains, which grow up 
Table 2. Predicted WR 118 parameters: color $V-J$; dereddened and reddenend visual magnitudes $V_{0}$ and $V$; distance $d$, bolometric luminosity $L_{\mathrm{bol}}$; radius $r_{\mathrm{in}}$ and density $\rho_{\mathrm{in}}$ at inner dust shell rim; and dust mass-loss rate $\dot{M}_{\text {dust }}$.

\begin{tabular}{lcccccccc}
\hline model & $V-J$ & $\begin{array}{c}V_{0} \\
(\mathrm{mag})\end{array}$ & $\begin{array}{c}V \\
(\mathrm{mag})\end{array}$ & $\begin{array}{c}d \\
(\mathrm{kpc})\end{array}$ & $\begin{array}{c}L_{\text {bol }} \\
\left(10^{4} L_{\odot}\right)\end{array}$ & $\begin{array}{c}r_{\text {in }} \\
\left(10^{3} R_{\odot}\right)\end{array}$ & $\begin{array}{c}\rho_{\text {in }} \\
\left(10^{-19} \mathrm{~g} / \mathrm{cm}^{3}\right)\end{array}$ & $\begin{array}{c}\dot{M}_{\text {dust }} \\
\left(10^{-7} M_{\odot} / \mathrm{yr}\right)\end{array}$ \\
\hline M 1 & 2.22 & 6.87 & 19.7 & 2.3 & 6.7 & 4.2 & 0.9 & 1.8 \\
M 2 & 1.68 & 6.33 & 19.1 & 1.8 & 5.6 & 3.0 & 1.2 & 1.3 \\
M 3 & 1.67 & 6.32 & 19.1 & 1.8 & 5.6 & 3.2 & 0.8 & 1.0 \\
M 1.1 & 2.15 & 6.46 & 20.5 & 1.9 & 5.5 & 3.4 & 0.9 & 1.2 \\
M 2.1 & 1.62 & 5.93 & 19.9 & 1.5 & 4.7 & 2.4 & 1.2 & 0.8 \\
\hline
\end{tabular}

from their small progenitors. In our double-sized grains models, we considered the combination of small grains $(a=0.05 \mu \mathrm{m})$ and large grains $(a=0.38 \mu \mathrm{m})$.

The temperature of the large grains is $\sim 1.5$ times lower than that of the small ones. Thus, if one increases the abundance of the large grains, $r_{\text {in }}$ has to be decreased to preserve the SED fits in the near-infrared domain. Although this even worsens the condition of dust-grain condensation in the corresponding models, the observed shape of the visibility curve clearly requires to include large grains in the dust shell of WR 118.

The decrease of the small grains' size does not affect the SED nor the visibility substantially, i.e. the grains with $a \leq 0.05 \mu \mathrm{m}$ behave similarly, in this respect, in the optical and IR domain. The increase of the relative abundance of the small grains mainly reflects in an increase of the emergent radiation's reddening in the optical region, and a decrease of the dust shell's albedo in the infrared. The latter leads to an essential change of the visibility's curvature in the "wrong" direction. Both these points define the abundance of small grains in the dust shell. However, in the case of WR 118, we have no accurate estimations of its brightness in the optical wavelength range, and thus cannot determine how much reddening we need. Calculations were performed adopting $A_{V}=12.8$ and 14, which leads to $J_{0} \approx 4.65$ and 4.31 (Allen et al. 1977; Williams et al. 1987), resp.

The increase of the large grains' size does not affect the SED and the $2.13 \mu \mathrm{m}$ visibility very much, either. However, the size of the large grains cannot be decreased significantly because the $2.13 \mu \mathrm{m}$ albedo of the Jäger et al. (1998) grains with $a=0.25 \mu \mathrm{m}$ is $\sim 0.27$ being already too low to allow a proper fit of the visibility. Besides double-sized grains models, we also calculated models with a power-law grain-size distribution of $n(a) \propto a^{-\gamma}(0.005 \mu \mathrm{m} \leq a \leq 0.6 \mu \mathrm{m})$ to investigate which exponent $\gamma$ is most appropriate. For all these models, $T_{\text {in }}$ is calculated for the smallest dust grains with $a=0.005 \mu \mathrm{m}$. This temperature exceeded that of the $a=0.05 \mu \mathrm{m}$ grains by $\sim 5 \%$.

\section{Results of calculations and discussion}

Figures 3-4 show the observed $2.13 \mu \mathrm{m}$ visibility of WR 118 and its SED corrected for interstellar extinction
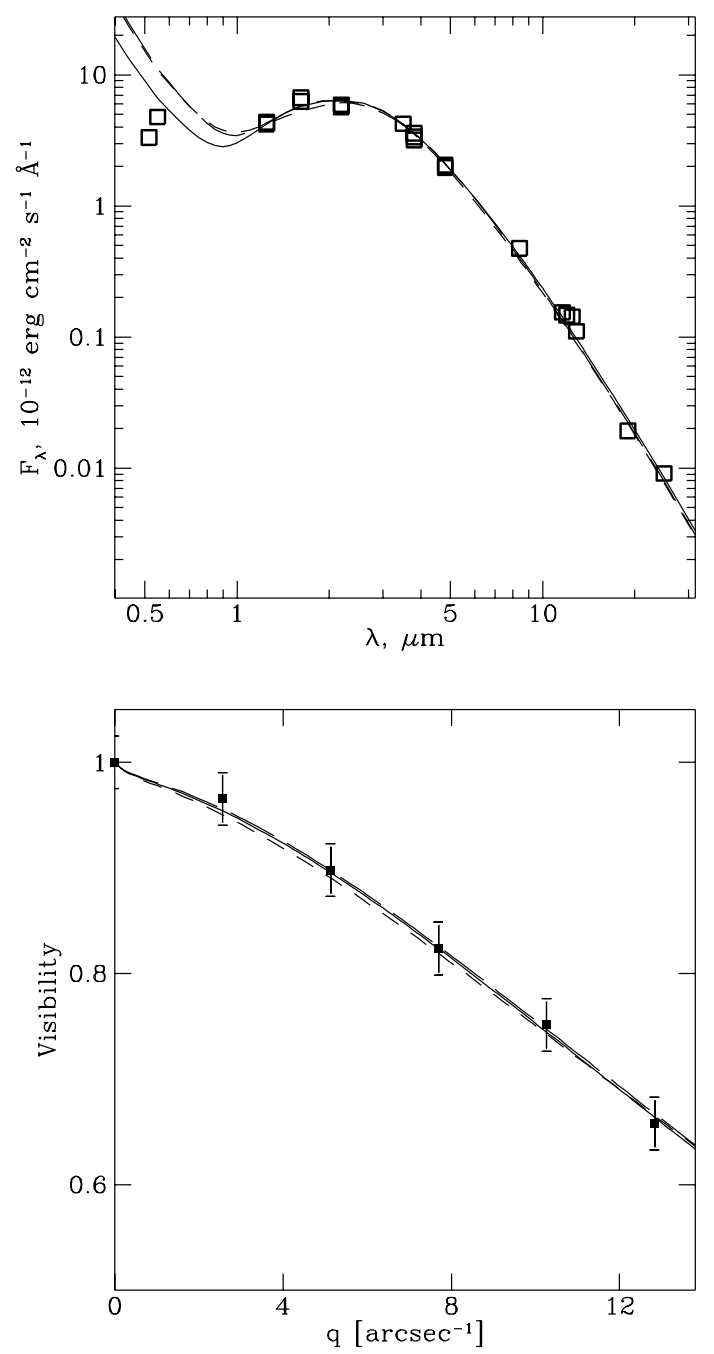

Fig. 3. Model SED corrected for interstellar extinction of $A_{V}=12.8$ and $2.13 \mu \mathrm{m}$ visibility function of WR 118 for the models M 1 (solid lines), M 2 (short-dashed lines), and M 3 (long-dashed lines) as given in Tables 1-2. The squares refer to the observations (see text).

of $A_{V}=12.8$ and 14 , respectively. The latter includes the data of Massey \& Conti (1983) ( $V$ band), Cohen \& Vogel (1978) ( $V$ band), Allen et al. (1977) ( $J H K L$ bands), Williams et al. (1987) ( $J H K L^{\prime} M$ [8.4], [11.6], [12.5], [12.9], and [19] bands) and color-corrected IRAS photometry (IRAS Science Team 1988) ([12] and [25] bands). 

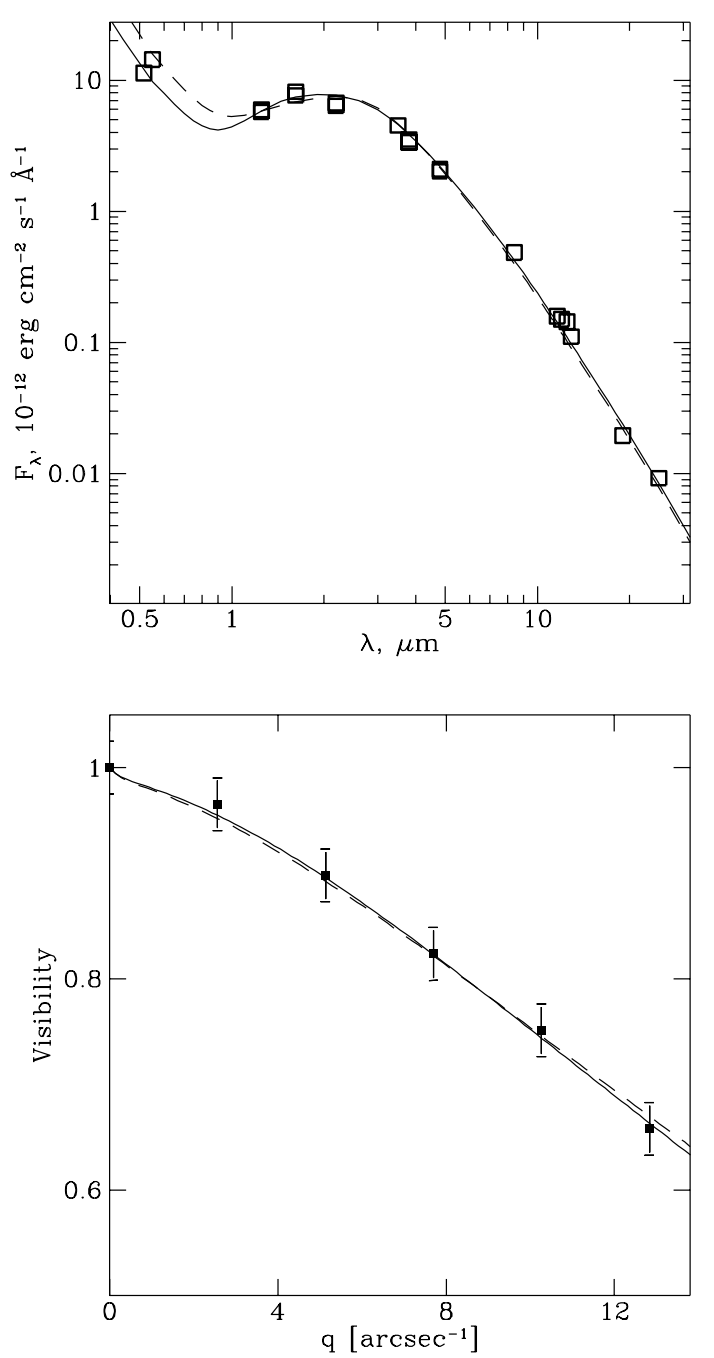

Fig. 4. Model SED corrected for interstellar extinction of $A_{V}=14.0$ and $2.13 \mu \mathrm{m}$ visibility function of WR 118 for the models M 1.1 (solid lines) and M 2.1 (short-dashed lines) as given in Tables 1-2. The squares refer to the observations (see text).

Figures 3 and 4 also show the $2.13 \mu \mathrm{m}$ visibility and SED of those 5 models, which proved to be best suited for the dust shell of WR 118 in the framework of spherical symmetry. Their parameters are given in Tables 1 and 2. For comparison, Williams et al. (1987) have found that the dust shell of WR 118 have the following parameters: $r_{\text {in }}=$ $260 R_{*}, Y_{\text {out }}=30, T_{\text {in }}=1410 \mathrm{~K}, \rho_{\text {in }}=4.9 \times 10^{-19} \mathrm{~g} / \mathrm{cm}^{-3}$ and $\dot{M}_{\text {dust }}=8.2 \times 10^{-7} M_{\odot} /$ yr.

The models M1, M 2, and M 3 refer to an interstellar extinction of $A_{V}=12.8$, and the models M 1.1 and M 2.1 to $A_{V}=14.0$. M 3 is the model with a power law grain-size distribution with $\gamma=3$. The other models refer to doublesized grains. In the latter case the relative abundance of $0.05 \mu \mathrm{m}$ grains in number $\left(n_{0.05}\right)$ and mass $\left(m_{0.05}\right)$ are given in Table 1, which also contains the optical depth of the dust envelope $\tau_{V}$ and its albedo at $2.13 \mu \mathrm{m}\left(A_{K}\right)$.

Regarding the models with the double-sized grains ( $a=0.05 \mu \mathrm{m}$ and $a=0.38 \mu \mathrm{m}$ ), the relative concentration of the $0.05 \mu \mathrm{m}$ grains is subject to some uncertainty, since
Table 3. Model dust-shell parameters for grain-size distributions with $n(a) \propto a^{-\gamma}$ and $0.005 \mu \mathrm{m} \leq a \leq 0.6 \mu \mathrm{m}$. $\tau_{V}$ is the optical depth at $0.55 \mu \mathrm{m} ; r_{\text {in }}, T_{\text {in }}$ and $\Theta_{\text {in }}$ are the radius, the temperature (refering to the smallest grains) and the angular diameter of the inner dust-shell rim, and $A_{K}$ is the $2.2 \mu \mathrm{m}$ dust-shell albedo.

\begin{tabular}{lcccccc}
\hline model & $\tau_{V}$ & $\begin{array}{c}r_{\text {in }} \\
\left(R_{*}\right)\end{array}$ & $\begin{array}{c}T_{\text {in }} \\
(\mathrm{K})\end{array}$ & $\gamma$ & $A_{K}$ & $\begin{array}{c}\Theta_{\text {in }} \\
(\mathrm{mas})\end{array}$ \\
\hline M 4 & 0.87 & 197 & 1610 & 3.5 & 0.29 & 19.2 \\
M 3 & 0.70 & 152 & 1760 & 3.0 & 0.36 & 17.5 \\
M 5 & 0.56 & 124 & 1900 & 2.5 & 0.38 & 17.1 \\
\hline
\end{tabular}

the small grains determine the reddening, which is not precisely known. A more accurate determination of this parameter demands a more precise estimation of the optical brightness of WR 118 at several wavelengths. However, the grain density at the inner boundary of the dust shell and the dust formation rate do not depend strongly on the adopted $A_{V}$ and relative grain concentration. Thus, the estimations given in Table 2 can be considered as reliable in the framework of a spherically symmetric dust shell model.

Furthermore, it turned out that a match of the SED at the region of $25 \mu \mathrm{m}$ can be improved if the exponent in the power law of the dust density distribution is $\alpha=2.1$ instead of 2. It remains open if this deviation from the uniform outflow is significant with respect to the observational and theoretical uncertainties. The same effect as increasing $\alpha$ can be reached by decreasing $Y_{\text {out }}$ to 300 . This is the lower limit for the dust thickness above which the $25 \mu \mathrm{m}$ flux is not affected any longer. However, such a sharp truncation of the outer parts of the dust shell looks less natural than, e.g., a gradual change of the mass-loss rate.

The M 1 and M 3 models have almost the same albedo $A_{K}$, and the M 2 and M 3 models have almost the same circumstellar (cst) reddening $\left(A_{V}-A_{J}\right) /\left.A_{V}\right|_{\text {cst }}$. It defines the similarity of the M1 and M 3 visibility curves and the similarity of corresponding SEDs (Fig. 1). The mass fraction of the grains with $a \geq 0.3 \mu \mathrm{m}$ in model M3 is $\sim 50 \%$.

Increasing the exponent $\gamma$ in the power-law grain-size distribution from 3.0 (M 3) to 3.5 (M 4) leads to a decrease of the albedo $A_{K}$ from 0.36 (Table 3 ) to 0.29 , and to a significant increase of the visibility curvature, and thus to a worse match of the observations. The strong dependence of the visibility curve's shape on the albedo is confirmed by model calculations with double-sized grains. On the other hand, the decrease of $\gamma$ to 2.5 does not change the albedo $A_{K}$ much (to only $\sim 0.38$ ) and correspondingly the visibility curve either (model M 5). However, then the model $V-J$ color decreases to 1.20 (1.67 for M 3) in contradiction to the observations. Moreover, the increase of the relative concentration of large grains enforces a decrease of $r_{\text {in }}$ to keep a good match of the SED in the near-infrared. This, in turn, leads to a corresponding increase of temperature 
at the inner rim, $T_{\text {in }}$, which is already very high (Table 3 ). Thus, from the models with power-law grain-size distributions the best one is that with $\gamma=3$.

If the wind of WR 118 is flattened due to some reason (due to stellar rotation, binarity), one can conclude from the circular symmetric shape of the two-dimensional visibility function, that one looks face-on at the flattened shell ("disk"). Williams et al. (1987) estimated the fraction of WR 118's luminosity absorbed by dust and re-radiated in the IR to be $\sim 71 \%$. If the star is not obscured by its dust disk, we estimate that this fraction should not be less than $50 \%$. This means that the dust disk should be optically thick and cover more than one half of the sphere. Such a dust shell would not look like a geometrically thin disk, but rather as a sphere with a hole in the line of sight, i.e. as a torus with a large opening angle. However, first we should have clear indications of such asphericities, before we introduce a more complex geometry to the dust shell model of WR 118. We note that van der Hucht (2001) suggested that all apparently single and persistently dustmaking WC9 stars may possibly owe their heated circumstellar dust signatures to colliding $\mathrm{WC}+\mathrm{OB}$ wind effects.

Recently, Veen et al. (1998) have attributed eclipselike variations of the optical brightness of the dusty Wolf-Rayet stars WR 103, WR 121 and WR 113 to the obscuration of starlight by dust clouds in the line of sight very close to the star, i.e. between the stellar surface and the permanent dust shell that is inferred from the IR excess. Moreover, they have estimated the sizes of the particles in the clouds to be of the order $0.1 \mu \mathrm{m}$. Crowther (1997) has discovered the visual fading of WR 104, accompanied by the disappearance of high-ionization spectral lines, and placed the dust clouds inside the permanent dust shell. Thus, the decrease of the dust shell's inner boundary proposed by our modelling does not look as extraordinary as it appears with respect to the even more hostile conditions for grain condensation.

\section{Conclusions}

We presented the first diffraction-limited $2.13 \mu \mathrm{m}$ speckle interferometric observations of the dusty Wolf-Rayet star WR 118 with 73 mas resolution. No evidence was found for any significant deviation of the dust shell from circular symmetry. Radiative transfer calculations were carried out to model the spectral energy distribution and our $2.13 \mu \mathrm{m}$ visibility function. The main conclusion of these calculations is that the grains in the permanent dust shell of the persistently dust-making Wolf-Rayet star WR 118 grow to the relatively large sizes $(a \geq 0.3 \mu \mathrm{m})$. They contribute more than $50 \%$ in total mass of the dust shell and rises its $2.13 \mu \mathrm{m}$ albedo up to $\sim 0.35$. The observations can be fitted either with a model containing double-sized grains with radii of $a=0.05 \mu \mathrm{m}$ and $0.38 \mu \mathrm{m}$ or, alternatively, by a model with a grain size distribution function $n(a) \sim a^{-3}$, with $a$ ranging between $0.005 \mu \mathrm{m}$ and $0.6 \mu \mathrm{m}$.
At the inner boundary of the dust shell, which has an angular diameter of $\Theta_{\text {in }}=(17 \pm 1)$ mas and is located at $r_{\text {in }}=(150 \pm 30) R_{*}$, the temperature of the smallest grains amounts to $1750 \mathrm{~K} \pm 100 \mathrm{~K}$ and the dust-shell density to $(1 \pm 0.2) \times 10^{-19} \mathrm{~g} / \mathrm{cm}^{3}$. Adopting a wind velocity of $V_{\text {wind }}=1200 \mathrm{~km} \mathrm{~s}^{-1}$, the dust formation rate is found to be $(1.3 \pm 0.5) \times 10^{-7} M_{\odot} /$ yr.

The existence of large grains in the dust shell leads to a revision of the previously assumed size of the inner dust-shell boundary. The inner dust-shell boundary is then located even closer to the star leading to higher grain temperatures. This, in turn, further worsens the conditions of grain condensation which are already extremely harsh in any case. The question how dust grains permanently condense under such conditions is still yet open (see Cherchneff et al. 2000 and references therein) but the existence of dust shells around Wolf-Rayet stars as in the case of WR 118 clearly shows its warrant urgency.

Acknowledgements. The observations were made with the SAO $6 \mathrm{~m}$ telescope, operated by the Special Astrophysical Observatory, Russia.

\section{References}

Allen, D. A., Hyland, A. R., Longmore, A. J., et al. 1977, ApJ, 217,108

Cherchneff, I., Le Teuff, Y. H., Williams, P. M., \& Tielens, A. G. G. M. 2000, A\&A, 357, 572

Cohen, M., \& Vogel, S. T. 1978, MNRAS, 185, 47

Crowther, P. A. 1997, MNRAS, 290, L59

Draine, B. T. 1985, ApJS, 57, 587

Egan, M. P., Leung, C. M., \& Spagna, G. F. 1988, Comput. Phys. Comm., 48, 271

IRAS Science Team 1988, Explanatory Supplement to the IRAS Point Source Catalogue, NASA

Ivezic, Z., \& Elitzur, M. 1997, MNRAS, 287, 1997

Jäger, C., Mutschke, H., \& Henning, T. 1998, A\&A, 332, 291

Labeyrie, A. 1970, A\&A, 6, 85

Leung, C. M. 1976, ApJ, 209, 75

Massey, P., \& Conti, P. S. 1983, PASP, 95, 440

Roche, P. F., \& Aitken, D. A. 1984, MNRAS, 208, 481

Schutte, W. A., van der Hucht, K. A., Whittet, D. C. B., et al. 1998, A\&A, 337, 261

van der Hucht, K. A. 2001, New Astron. Rev., 45, 135

van der Hucht, K. A., Cassinelli, J. P., \& Williams, P. M. 1986, A\&A, 168, 111

van der Hucht, K., Williams, P. M., Morris, P. W., et al. 1996, A\&A, 315, L193

Veen, P. M., van Genderen, A. M., van der Hucht, K. A., et al. 1998, A\&A, 329, 199

Williams, P. M., van der Hucht, K. A., \& The, P. S. 1987, A\&A, 182, 91

Williams, P. M., van der Hucht, K. A., \& Morris, P. W. 1998, Ap\&SS, 255, 169

Williams, P. M., \& van der Hucht, K. A. 2000, MNRAS, 314,23 